\title{
Suppression of Musashi-2 by the small compound largazole exerts inhibitory effects on malignant cells
}

\author{
MIN WANG $^{1-3^{*}}$, XIAO-YAN SUN ${ }^{1 *}$, YONG-CHUN ZHOU ${ }^{4}$, KUO-JUN ZHANG $^{5}$, YONG-ZHI LU ${ }^{6}$, \\ JINSONG LIU ${ }^{6}$, YUN-CHAO HUANG ${ }^{4}$, GUI-ZHEN WANG ${ }^{1,2}$, SHENG JIANG ${ }^{5}$ and GUANG-BIAO ZHOU ${ }^{1,2}$ \\ ${ }^{1}$ State Key Laboratory of Membrane Biology, Institute of Zoology, Chinese Academy of Sciences, Beijing 100101; \\ ${ }^{2}$ State Key Laboratory of Molecular Oncology, National Cancer Center/National Clinical Research Center for \\ Cancer/Cancer Hospital, Chinese Academy of Medical Sciences and Peking Union Medical College, Beijing 100021; \\ ${ }^{3}$ University of Chinese Academy of Sciences, Beijing 100049; ${ }^{4}$ Department of Thoracic Surgery, \\ The Third Affiliated Hospital of Kunming Medical University, Kunming, Yunnan 650106; ${ }^{5}$ State Key Laboratory of \\ Natural Medicines, and Department of Medicinal Chemistry, School of Pharmacy, China Pharmaceutical University, \\ Nanjing, Jiangsu 210009; ${ }^{6}$ State Key Laboratory of Respiratory Disease, Guangzhou Institutes of \\ Biomedicine and Health, Chinese Academy of Sciences, Guangzhou, Guangdong 510530, P.R. China
}

Received August 8, 2019; Accepted January 30, 2020

DOI: 10.3892/ijo.2020.4993

\begin{abstract}
RNA-binding protein Musashi-2 (MSI2) serves as a regulator of numerous pivotal biological processes associated with cancer initiation, development and resistance to treatment, and may represent a promising drug target. However, whether MSI2 inhibition is of value in antitumor treatment remains to be determined. The present study demonstrated that MSI2 was upregulated in non-small cell lung cancer (NSCLC) and was inversely associated with the clinical outcome of the patients. Molecular docking analysis demonstrated that the small compound largazole binds to and may be a potential inhibitor of MSI2. Largazole markedly decreased the protein and mRNA levels of MSI2 and suppressed its downstream mammalian target of rapamycin signaling pathway. Largazole also inhibited the proliferation and induced apoptosis of NSCLC and chronic myeloid leukemia (CML) cells (including bone marrow mononuclear cells harvested from CML patients). These results indicate that MSI2 is an emerging therapeutic target for NSCLC and CML, and the MSI2 inhibitor largazole may hold promise as a treatment for these malignancies.
\end{abstract}

Correspondence to: Dr Guang-Biao Zhou, State Key Laboratory of Molecular Oncology, National Cancer Center/National Clinical Research Center for Cancer/Cancer Hospital, Chinese Academy of Medical Sciences and Peking Union Medical College, 17 Pan-Jia-Yuan-Nan-Li, Chaoyang District, Beijing 100021, P.R. China E-mail: gbzhou@cicams.ac.cn

*Contributed equally

Key words: Musashi-2, inhibitor, non-small cell lung cancer, chronic myeloid leukemia, largazole

\section{Introduction}

Lung cancer is the leading cause of cancer-related mortality worldwide, resulting in 1.8 million deaths annually (1). Lung cancer is mainly subdivided into non-small cell lung cancer (NSCLC) and small-cell lung cancer, whereas $~ 85 \%$ of lung cancers are NSCLC, which includes three major histological subtypes: Lung adenocarcinoma (LUAD), lung squamous cell carcinoma (LUSC), and large-cell lung cancer (2). Over the past two decades, the use of targeted therapies and immunotherapies has achieved survival benefits in a proportion of the patients $(3,4)$. However, the 5 -year survival rate for lung cancer of all stages combined was only $18.6 \%$ in $2019(5,6)$; therefore, there is an urgent need to identify new molecular targets in order to develop novel therapies and improve patient outcomes.

RNA-binding proteins (RBPs) are crucial regulators of RNA stability, splicing and translation, and play key roles in the pathological processes underlying multiple diseases, including cancer $(7,8)$. The Musashi family of RBPs comprises Musashi-1 (MSI1) and Musashi-2 (MSI2), which play complementary as well as independent roles in stem cells $(9,10)$. MSI2 belongs to the class A/B heterogeneous nuclear ribonucleoproteins (hnRNPs) and contains two tandem RNA recognition motifs and a carboxyl-terminal poly-A-binding protein association domain $(10,11)$. Recently, MSI2 was suggested to be a potential oncoprotein regulating cancer initiation, development, and resistance to treatment (12). MSI2 has been found to be increased in chronic myeloid leukemia (CML) (13), acute myeloid leukemia (AML) (14) and several types of solid tumors, including colorectal $(15)$, lung $(16,17)$, breast $(18)$, cervical $(11,19)$, and pancreatic cancer (20), as well as several other cancers (21-23). Overexpression of MSI2 promotes the proliferation, invasion and metastasis of pancreatic (20), cervical (19) and esophageal squamous cell carcinoma cells (21), and induces resistance to paclitaxel in ovarian cancer cells (24). Knockdown of 
MSI2 inhibits cell proliferation, invasion and metastasis in NSCLC $(16,17)$ and leukemia $(25,26)$, and sensitizes AML cells to treatment with daunorubicin (26). These findings suggest that MSI2 may be a promising therapeutic target in cancer. Two small compounds, gossypolone and Ro 08-2750, were shown to be able to inhibit MSI1 and MSI2 $(27,28)$. However, the effects of MSI2 inhibition on lung cancer cells remain to be investigated.

The aim of the present study was to investigate the potential of MSI2 as a drug target for lung cancer therapy, and identify natural compounds targeting MSI2 and evaluate their antitumor activity. The expression of MSI2 was examined in NSCLC cell lines and human lung cancer specimens, and it was investigated whether its expression is associated with prognosis. Furthermore, the effects of largazole, a marine natural cyclic depsipeptide extracted from a cyanobacterium of the genus Symploca (29), on the expression of MSI2 and its downstream mammalian target of rapamycin (mTOR) signaling pathway, cancer cell proliferation and apoptosis were investigated, in order to determine whether largazole may achieve clinical benefits through MSI 2 inhibition.

\section{Materials and methods}

Patient samples. The present study was approved by the Institutional Review Board of the Institute of Zoology, Chinese Academy of Sciences and the Third Affiliated Hospital of Kunming Medical University; all tissue samples were obtained with written informed consent from the patients or their families. Tumor and adjacent normal lung tissues were obtained between June and November 2011 from 6 patients with previously untreated NSCLC. These included two men and four women, with a mean age of 58 years (range, $41-68$ years). The samples were immediately frozen in liquid nitrogen following surgical resection. The expression of MSI2 at the mRNA level was assayed in The Cancer Genome Atlas (TCGA) transcriptome database containing 1,053 NSCLC tumor samples and 109 normal lung specimens. The survival curve was estimated by the Kaplan-Meier method and log-rank test using the Online Survival Analysis Software (30) (http://kmplot. com/analysis/index.php?p=service\&cancer=lung).

Reagents and antibodies. Largazole was synthesized by our chemistry laboratory (31). The purity of largazole was 98\% (determined by reverse-phase high-performance liquid chromatography). PS-341 was obtained from Millennium Pharmaceuticals and chloroquine (CQ) was purchased from Sigma-Aldrich; Merck KGaA. The antibodies used included rabbit anti-human MSI2 (cat. no. ab76148, Abcam; 1:1,000 for western blotting), rabbit anti-human S6K (cat. no. 9202, 1:1,000 for western blotting), rabbit anti-human p-S6K (Thr389) (cat. no. 9205, 1:1,000 for western blotting), rabbit anti-human p-S6 ribosomal protein (Ser240/244) (cat. no. 5364, 1:1,000 for western blotting), rabbit anti-human PARP (cat. no. 9542, 1:1,000 for western blotting), rabbit anti-human p-4E-BP1 (Ser65) (cat. no. 9451, 1:1,000 for western blotting) from Cell Signaling Technology, Inc., mouse anti-human actin (cat. no. A5441, Sigma-Aldrich; Merck KGaA; 1:5,000 for western blotting), rabbit anti-human p-Akt (Ser473) (cat. no. sc-7985-R, 1:500 for western blotting) and rabbit anti-human Akt (cat. no. sc-8312, 1:500 for western blotting) from Santa Cruz Biotechnology, Inc.

Cell culture. The human normal lung epithelial cell line $16 \mathrm{HBE}$ was obtained from Clonetics. The C57BL/6 murine Lewis lung carcinoma (LLC) cell line, the human leukemic cell line K562, and the NSCLC cell lines A549, H460, H520 and H1975 (harboring EGFR-L858R/T790M mutations) were obtained from the American Type Culture Collection. The 32Dcl3-Bcr-Abl-T315I (32D-BA-T315I) cell line was obtained by stably infecting $32 \mathrm{Dcl} 3$ cells with the MSCV-Bcr-Abl-T315I-IRES/GFP (Bcr-Abl-T315I/GFP) retroviral transducing vector, which was kindly provided by Dr Warren Pear (University of Pennsylvania). The pSRalpha plasmid constructs containing the wild-type and T315I mutant cDNAs of the Bcr-Abl tyrosine kinase were provided by Dr Brian Druker (Oregon Health \& Science University). Cells expressing 32Dcl3-Bcr-Abl (32D-BA), 32D-BA-T315I and the imatinib-resistant cell line K562R were established and kept in our laboratory (32). All cell lines were cultured according to the recommended protocols.

Cell viability and apoptosis assay. Cell viability was determined by the Cell Counting Kit-8 (CCK-8; Dojindo Molecular Technologies, Inc.) assay according to the manufacturer's protocol: Cell suspensions $(100 \mu \mathrm{l})$ were added into each well of 96-well plates at a density of 5,000 cells/well, the cells were then exposed to the indicated concentrations of largazole for 24 or $48 \mathrm{~h}, 10 \mu \mathrm{l}$ of CCK- 8 reagent was added to each well, and the cells were incubated at $37^{\circ} \mathrm{C}$ for $1-2 \mathrm{~h}$. The absorbance was measured at $450 \mathrm{~nm}$ using a microplate reader (Bio-Tek Instruments, Inc.). Cell apoptosis was analyzed using the Annexin V-FITC Apoptosis Detection kit (BD Biosciences) according to the manufacturer's instructions. The cells were treated with the indicated concentrations of largazole for 48 $\mathrm{h}$ and collected, resuspended in Annexin $\mathrm{V}$ binding buffer, incubated with Annexin V-FITC and propidium iodide (PI) for $15 \mathrm{~min}$ at room temperature in the dark, and tested by flow cytometry using a FACS Calibur flow cytometer (Becton, Dickinson and Company). FACS data were analyzed by BD CellQuest ${ }^{\mathrm{TM}}$ Pro 6.0 software (Becton, Dickinson and Company).

Soft agar colony formation assay. Cells were treated with the indicated concentrations of largazole for $12 \mathrm{~h}$, then counted and resuspended in RPMI-1640 containing 10\% fetal bovine serum (Gibco; Thermo Fisher Scientific, Inc.) and $0.3 \%$ low-melting-point agarose (Amresco), and plated on the bottom layer in a $35-\mathrm{mm}$ plate containing $0.6 \%$ agarose; the experiment was performed in triplicate. After 12 days of culture, the colonies were stained with $0.005 \%$ crystal violet solution for $30 \mathrm{~min}$ at room temperature (Sigma-Aldrich; Merck $\mathrm{KGaA}$ ) and counted.

Western blot analysis. For western blot analysis in cultured cells, NSCLC and CML cells were treated with the indicated protocols, then harvested and lysed in RIPA buffer containing $50 \mathrm{mM}$ Tris (pH 7.4), $150 \mathrm{mM} \mathrm{NaCl}, 0.1 \%$ sodium dodecyl sulfate, $1 \%$ sodium deoxycholate, $1 \%$ Triton $\mathrm{X}-100,1 \mathrm{mM}$ EDTA, $1 \mathrm{mM} \mathrm{Na}_{3} \mathrm{VO}_{4}, 1 \mathrm{mM} \mathrm{NaF}, 1 \mathrm{mM}$ PMSF and protease 

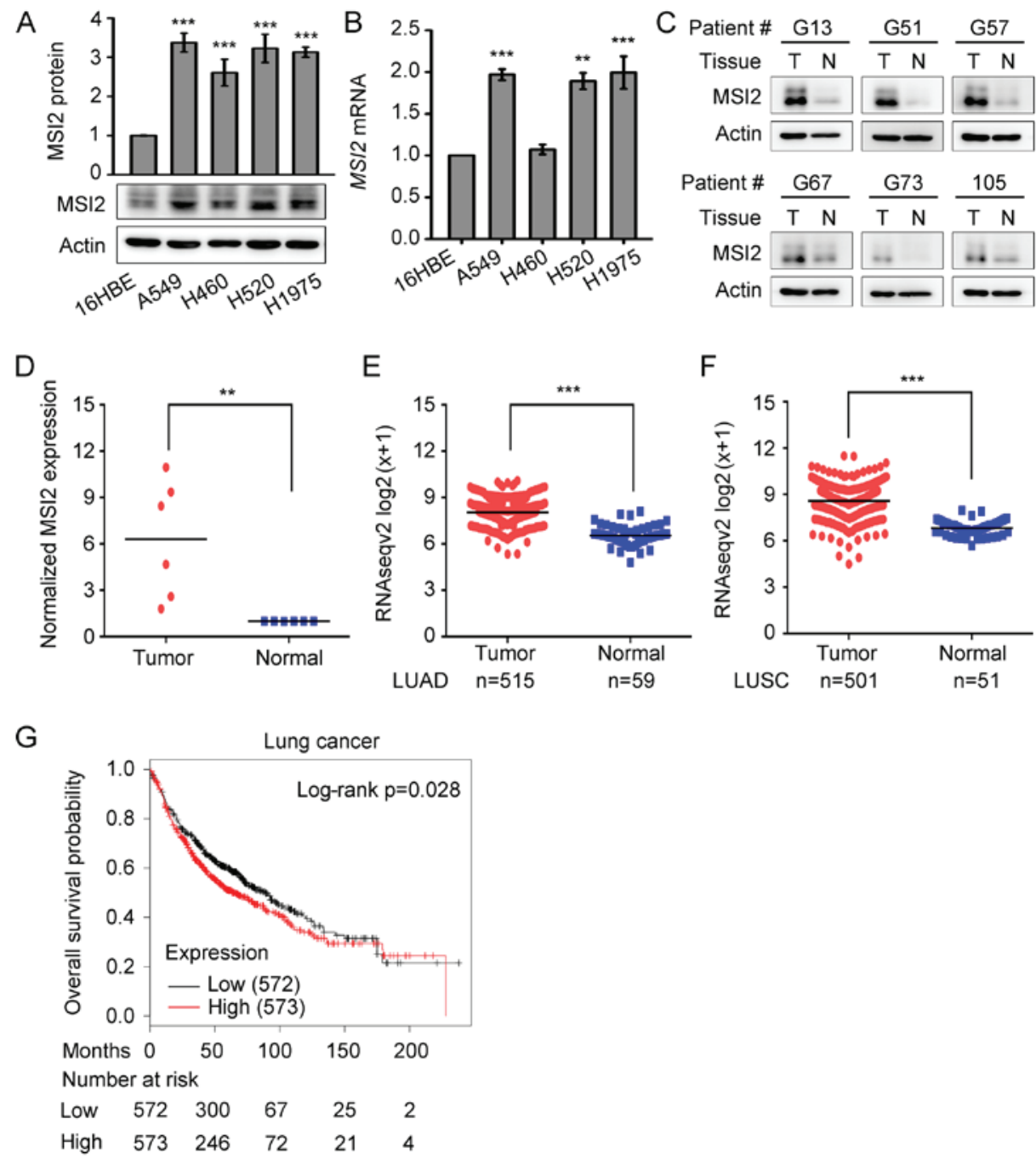

Figure 1. MSI2 is upregulated in lung cancer and is inversely associated with prognosis. (A) The protein levels of MSI2 in human normal lung epithelial 16HBE cells and lung cancer cell lines were detected by western blotting. Actin was used as a loading control. The protein levels of MSI2 were semi-quantified using NIH ImageJ software. Repeated number, $\mathrm{n}=3 .{ }^{* * *} \mathrm{P}<0.001$, difference vs. $16 \mathrm{HBE}$ cells. (B) The mRNA levels of MSI2 were quantified by quantitative PCR and normalized to $16 \mathrm{HBE}$ cells. Repeated number, $\mathrm{n}=3 .{ }^{* *} \mathrm{P}<0.01,{ }^{* * *} \mathrm{P}<0.001$, difference vs. $16 \mathrm{HBE}$ cells. (C) Western blot analyses of lysates of tumor and adjacent normal lung tissues obtained from lung cancer patients. Actin was used as a loading control. (D) Relative densitometric quantification of MSI2 protein levels detected in (C). ${ }^{* *} \mathrm{P}<0.01$. (E and F) MSI2 expression in LUAD and LUSC patients in TCGA dataset. ${ }^{* * *} \mathrm{P}<0.001$. (G) Kaplan-Meier analysis of overall survival of lung cancer patients stratified by the expression levels of MSI2. Numbers of patients and log-rank P-value are shown. MSI2, Musashi-2; LUAD, lung adenocarcinoma; LUSC, lung squamous cell carcinoma; TCGA, The Cancer Genome Atlas.

inhibitor cocktail. For western blot analysis in tissue specimens, frozen tissues were ground in liquid nitrogen-cooled mortar, tissue powder was lysed on ice in RIPA buffer. The lysates were centrifuged at $12,000 \mathrm{xg}$ for $10 \mathrm{~min}$ at $4^{\circ} \mathrm{C}$, the supernatant was dissolved with $5 \mathrm{X}$ sample loading buffer containing $250 \mathrm{mM}$ Tris- $\mathrm{HCl}$ ( $\mathrm{pH} 6.8$ ), $10 \%$ sodium dodecyl sulfate, $50 \%$ glycerol, $2.5 \%$ bromophenol blue, and $5 \% \beta$-mercaptoethanol, and boiled for $5 \mathrm{~min}$. Equivalent amounts of protein (30 $\mu \mathrm{g} /$ lane) were separated by $10-15 \%$ sodium dodecyl sulfate polyacrylamide gel electrophoresis and transferred to nitrocellulose membranes. The membranes were blocked with $5 \%$ non-fat milk and incubated with the indicated primary and corresponding secondary antibodies, i.e., horseradish peroxidase-conjugated anti-mouse (cat. no. 115-035-003, Jackson ImmunoResearch Laboratories, Inc.; 1:10,000) or anti-rabbit (cat. no. 111-035-003, Jackson ImmunoResearch Laboratories, Inc.; 1:10,000). Immunoreactive bands were visualized by using Luminescent Image Analyzer LSA 4000
(GE Healthcare). Densitometry analysis was performed using ImageJ software (version 1.4.3.67; National Institutes of Health).

Reverse transcription-quantitative PCR (RT-qPCR) analysis. Total RNA was prepared with TRIzol reagent (Invitrogen; Thermo Fisher Scientific, Inc.) according to the manufacturer's instructions. RT was carried out using a First Strand cDNA Synthesis kit (Fermentas; Thermo Fisher Scientific, Inc.). The RT steps were as follows: $25^{\circ} \mathrm{C}$ for $10 \mathrm{~min}, 42^{\circ} \mathrm{C}$ for $60 \mathrm{~min}$ and $70^{\circ} \mathrm{C}$ for 10 min. qPCR was performed in $\mathrm{CFX}^{\mathrm{TM}} 96$ Real Time System (Bio-Rad Laboratories, Inc.) using SYBR Premix Ex Taq $^{\mathrm{TM}}$ (Takara Biotechnology, Inc.) according to the manufacturer's protocol. The thermocycling conditions of the qPCR step were as follows: $95^{\circ} \mathrm{C}$ for 5 min followed by 40 cycles of $95^{\circ} \mathrm{C}$ for $15 \mathrm{sec}$ and $60^{\circ} \mathrm{C}$ for $30 \mathrm{sec}$. The primer sequences were as follows: Human GAPDH, forward 5'-GGAGCGAGA TCCCTCCAAAA-3' and reverse 5'-GGCTGTTGTCATACT 
A

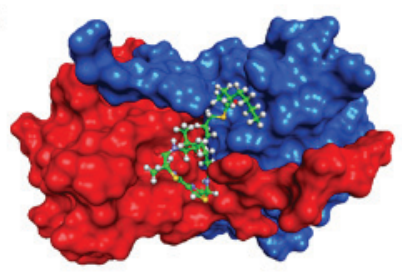

B

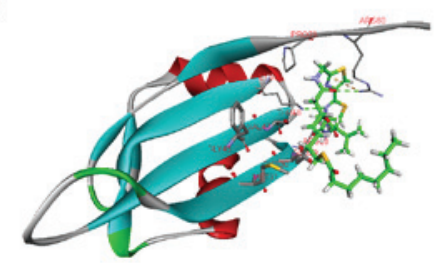

C

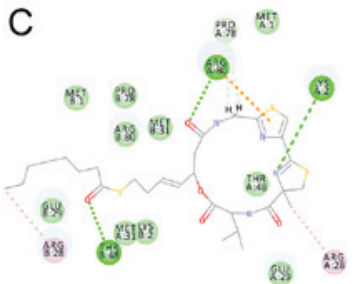

Interactions

$\square$ van der Waals

Conventional Hydrogen Bond

$\checkmark$ Carbon Hydrogen Bond

- Pi-Cation

$\square$ Alkyl
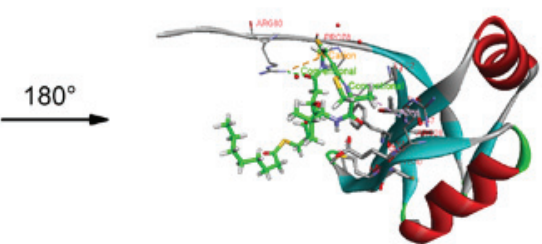

Figure 2. Docking analysis between largazole and the human MSI2 protein. The preferable pose was selected for analysis. (A) The binding pocket surfaces are shown. (B) MSI2 RRM1 protein structure is shown as cartoon and the docked pose is shown as sticks (bonds) and spheres (atoms). (C) 2D diagram of largazole-MSI2 interaction. MSI2, Musashi-2; RRM1; RNA-recognition motif 1.

TCTCATGG-3'; human MSI2, forward 5'-GTTATCTGCGAA CACAGTAGTG-3' and reverse 5'-ACCCTCTGTGCCTGT TGGTAG-3'.

Molecular docking analysis. The structure of largazole was prepared by ChemBioDraw Ultra 14.0 (PerkinElmer, Inc.) and was converted to 3D model via the Ligprep module in Schrödinger Maestro 10.5 (Schrödinger LLC). We performed docking studies with a reported human MSI2 structure (28). The docking procedure was performed by glide docking in Maestro with a precision level of SP (Standard Precision). The docking results were analyzed by Biovia Discovery Studio 2016 client (Dassault Systèmes Biovia Cc).

Statistical analysis. The results are expressed as mean \pm standard deviation. Two-tailed Student's t-test was used to determine the statistical significance between two groups. One-way ANOVA with Dunnett's or Bonferroni's post hoc test was used for the comparison of multiple groups. $\mathrm{P}<0.05$ was considered to indicate statistically significant differences.

\section{Results}

MSI2 is upregulated in lung cancer and is inversely associated with prognosis. To assess the MSI2 expression in lung cancer, the expression levels of MSI2 in normal human lung epithelial 16HBE cells and four lung cancer cell lines (A549, H460, H520 and H1975) were first analyzed by western blotting and qPCR. Compared with the normal lung epithelial 16HBE cells, the lung cancer cell lines exhibited elevated protein and mRNA levels of MSI2 (Fig. 1A and B). The expression of MSI2 was further examined in lung cancer tissues from 6 patients with NSCLC. As shown in Fig. 1C and D, the protein levels of MSI2 were significantly increased in lung cancer tissues compared with those in adjacent normal lung tissues. Since the sample size of this study was small, we explored the expression levels of MSI2 in TCGA level 3 IlluminaHiseq RNAseqV2 transcriptome database containing 1,053 NSCLC tumor samples and 109 normal lung specimens. This analysis revealed that MSI2 expression was significantly higher in LUADs $(n=515)$ and LUSCs $(n=501)$ compared with that in normal lung tissues (Fig. 1E and F). To further investigate the correlation between MSI2 expression and the survival of lung cancer patients, Kaplan-Meier analysis was performed using the online cancer survival analysis database (http://kmplot.com/analysis/) (30). As shown in Fig. 1G, lung cancer patients with higher expression of MSI2 had poorer overall survival (OS) compared with those with lower expression of MSI2. The data mentioned above suggest that MSI2 is involved in the pathogenesis of lung cancer and may be a useful therapeutic target.

MSI2 is targeted by the small compound largazole. Largazole is a natural macrocyclic depsipeptide isolated from the marine cyanobacterium Symploca sp. (33). Our team has successfully synthesized this compound, as well as some analogues, and demonstrated its potent antitumor activity in certain cancers (including lung cancer) at $0.01-1 \mu \mathrm{M}(31,34)$. By molecular docking analysis, we herein demonstrated that largazole was able to bind to MSI2. A crystal structure of the apo human MSI2 RNA-recognition motif 1 (RRM1) at $1.7 \AA$ resolution was recently obtained (28). Docking studies with this structure were performed and it was reported that largazole can be docked into the dimer structure of MSI2 with most of the compound interacting with one of the MSI2 chains in 6DBP (chain A, red), while the thioester residue falls into the cave formed by residues in chain B (blue; Fig. 2A). The backbone part of largazole mainly interacts with the $\beta$-sheets (Arg28-Met31, Phe46-Phe49) of MSI2 (Fig. 2B). As shown in Fig. 2B and $\mathrm{C}$, the valine residue forms an alkyl-alkyl bond with the side chain of Glu29. The methyl group attached at 4,5-dihydrothiazole forms an alkyl bond with Arg28. A hydrogen bond forms between Lys 2 and nitrogen in 4,5-dihydrothiazole. As regards the thiazole ring, a pi-cation interaction forms with the side chain of Arg80. Furthermore, the guanidine group of Arg80 forms a hydrogen bond with the carbonyl group of the HoSer residue. The predicted binding energy of this docking model is $-30.2752 \mathrm{kcal} / \mathrm{mol}$.

Largazole inhibits the expression of MSI2. The data presented above prompted us to determine whether largazole inhibits 


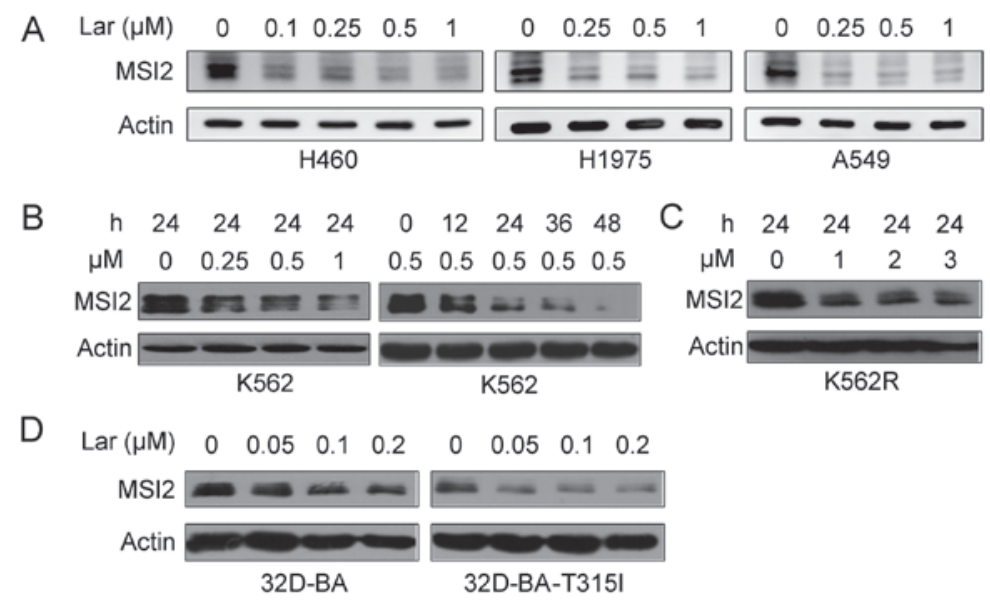

Figure 3. Largazole inhibits the expression of MSI2. (A) NSCLC cells were treated with the indicated concentrations of largazole for $24 \mathrm{~h}$, and the expression levels of MSI2 were analyzed by western blotting. Actin was used as a loading control. (B) K562 cells were treated with the indicated concentrations of largazole for indicated times, and the expression levels of MSI2 were analyzed by western blotting. (C) K562R cells were treated with the indicated concentrations of largazole for $24 \mathrm{~h}$, followed by western blot analysis with the indicated antibodies. (D) 32Dcl3 cells expressing wild-type Bcr-Abl (BA) or Bcr-Abl with T315I mutation (BA-T315I) were treated with the indicated concentrations of largazole for $24 \mathrm{~h}$, and the expression levels of MSI2 were analyzed by western blotting. MSI2, Musashi-2; NSCLC, non-small cell lung cancer.
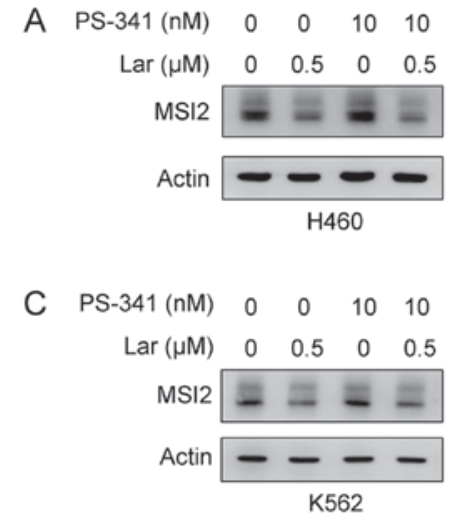

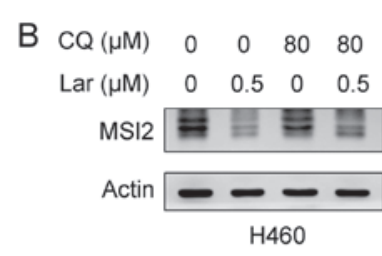

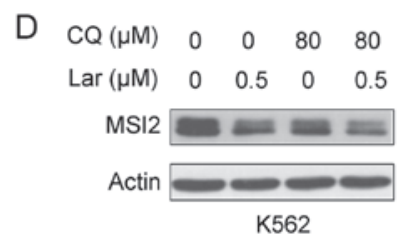
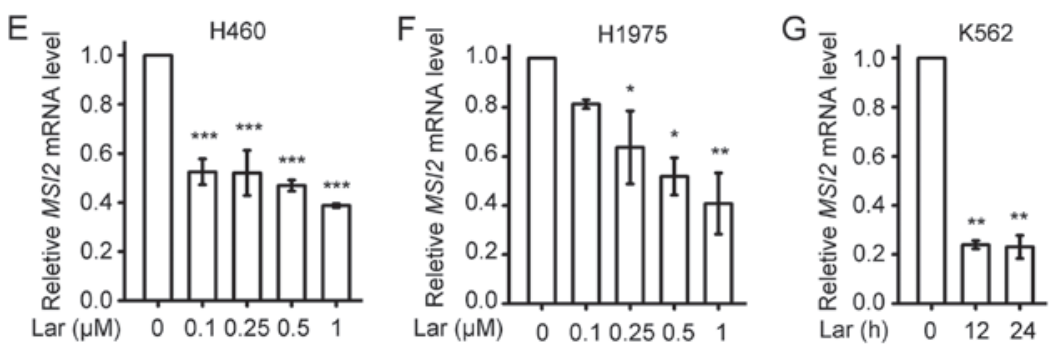

Figure 4. Largazole induces downregulation of MSI2 at the mRNA level. (A-D) H460 and K562 cells were pretreated with or without $10 \mathrm{nM}$ PS-341/80 $\mu \mathrm{M}$ $\mathrm{CQ}$ for $2 \mathrm{~h}$, followed by largazole treatment at $0.5 \mu \mathrm{M}$ for $24 \mathrm{~h}$. The cells were harvested and subjected to western blot analysis using the indicated antibodies. Actin was used as a loading control. (E and F) H460 and H1975 cells were treated with the indicated concentrations of largazole for 24 h, and the mRNA levels of MSI2 were quantified by quantitative PCR (qPCR). Repeated number, $\mathrm{n}=3$. ${ }^{*} \mathrm{P}<0.05,{ }^{* *} \mathrm{P}<0.01$ and ${ }^{* * *} \mathrm{P}<0.001$, difference vs. control group. (G) K562 cells were treated with $0.5 \mu \mathrm{M}$ largazole for $12-24 \mathrm{~h}$, and the mRNA levels of $M S I 2$ were quantified by qPCR. ${ }^{* *} \mathrm{P}<0.01$, difference vs. control group. MSI2, Musashi-2; CQ, chloroquine.

the expression of MSI2. Western blot analysis demonstrated that treatment with indicated concentrations of largazole for $24 \mathrm{~h}$ drastically inhibited the expression of MSI2 in human lung cancer H460, H1975 and A549 cells (Fig. 3A). MSI2 plays a critical role in CML progression $(13,14)$. The effects of this compound were tested on CML cells, and it was found that largazole also repressed the protein expression of MSI2 in K562 CML cells in a concentration- and time-dependent manner (Fig. 3B). Largazole decreased the expression of MSI2 in the K562R cell line, which is resistant to the Bcr-Abl tyrosine kinase inhibitor imatinib (Fig. 3C). In a pair of 32Dcl3 murine cell lines stably expressing wild-type or T315I mutant Bcr-Abl, namely 32D-BA and 32D-BA-T315I cells, treatment with largazole at $0.05-0.2 \mu \mathrm{M}$ for $24 \mathrm{~h}$ inhibited MSI 2 expression (Fig. 3D).

Largazole decreases MSI2 at the mRNA level. To further understand the mechanism underlying largazole-induced 

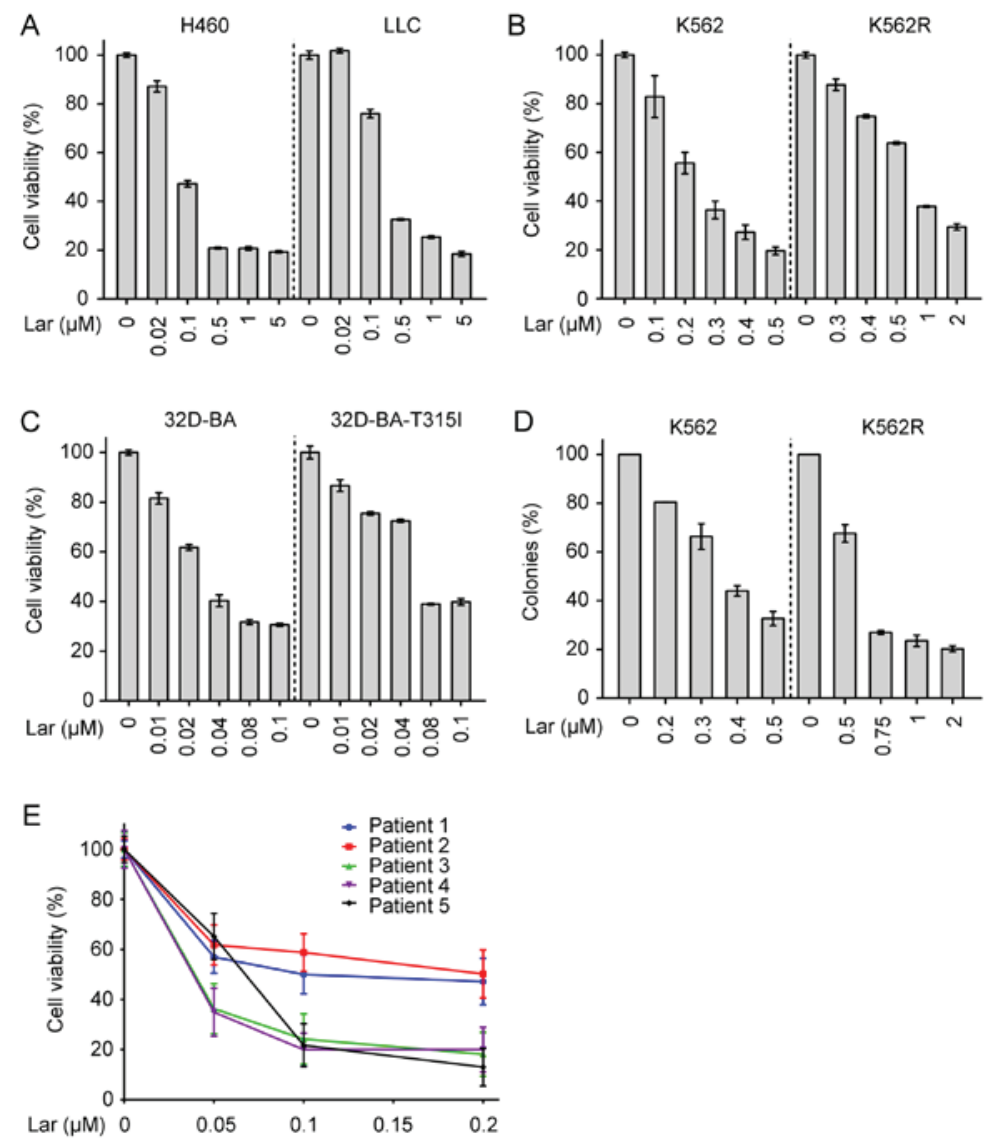

Figure 5. Largazole inhibits the proliferation of cancer cell lines and mononuclear cells harvested from patients with CML. Repeated number, $\mathrm{n}=3$. (A) NSCLC cells were treated with the indicated concentrations of largazole for $48 \mathrm{~h}$, and cell viability was determined by the CCK- 8 assay and is presented as relative viability to the control group. (B and C) CML cells were treated with the indicated concentrations of largazole for $24 \mathrm{~h}$, cell viability was determined by the CCK-8 assay and is presented as relative viability to the control group. (D) Colony formation assay was performed in K562 and K562R cells treated with the indicated concentrations of largazole. (E) The CCK-8 assay was used to assess the antiproliferative effect of largazole on mononuclear cells that were isolated from the bone marrow of 5 patients with CML. MSI2, Musashi-2; CML, chronic myeloid leukemia; CCK-8, Cell Counting Kit-8.

downregulation of MSI2, the proteasome inhibitor PS-341 and lysosome inhibitor CQ were used. As shown in Fig. 4A-D, pretreatment with PS-341 or CQ could not block largazole-induced downregulation of MSI2 in H460 and K562 cells. Furthermore, treatment of H460 and H1975 cells with largazole for $24 \mathrm{~h}$ also decreased the mRNA levels of MSI2 in a concentration-dependent manner (Fig. 4E and F). Treatment of K562 cells with $0.5 \mu \mathrm{M}$ largazole for 12-24 h markedly reduced the mRNA levels of MSI2 (Fig. 4G).

Largazole inhibits proliferation and induces apoptosis of cancer cells. Our previous study has demonstrated that largazole markedly inhibits the proliferation of multiple lung cancer cell lines and induces apoptosis of lung cancer A549 cells (34). The present study demonstrated that treatment with largazole significantly repressed the proliferation of $\mathrm{H} 460$, LLC, K562, K562R, 32D-BA and 32D-BA-T315I cells in a dose-dependent manner (Fig. 5A-C). Largazole significantly suppressed the colony-forming ability of K562 and K562R cells, as assessed by a soft agar colony formation assay (Fig. 5D). Of note, largazole markedly inhibited the proliferation of mononuclear cells that were isolated from the bone marrow of 5 patients with CML (Fig. 5E).

Largazole-induced apoptosis was investigated by Annexin V-FITC/PI double staining, and the results demonstrated that treatment of K562 and K562R cells with largazole at 1 and $2 \mu \mathrm{M}$ for $48 \mathrm{~h}$ significantly increased the percentage of Annexin $\mathrm{V}^{+}$apoptotic cells (Fig. 6A and B). Western blot analysis revealed that largazole caused the cleavage of poly(ADP-ribose) polymerase (PARP) in H460, H1975 (Fig. 6C), K562, K562R (Fig. 6D) and 32D-BA-T315I cells (Fig. 6E), indicating activation of the apoptosis effector caspase-3.

Largazole inhibits the downstream mTOR signaling pathway of MSI2. One of the main oncogenic pathways downstream of MSI2 is mTOR complex 1 (mTORC1), which becomes activated upon MSI2 binding to the tumor suppressor phosphatase and tensin homolog $(15,35)$. Accumulating evidence indicates that the mTOR signaling pathway plays a key role in regulating cell metabolism, growth, proliferation and survival $(36,37)$. The effects of largazole on the mTOR signaling pathway were examined. Western blot analysis demonstrated that largazole repressed the phosphorylation of S6K and 4E-BP1, the two best characterized downstream effector molecules of mTORC1 (38), in a concentration-dependent manner in H460, H1975 and A549 cells (Fig. 7A-C). Largazole also inhibited the phosphorylation of S6, a substrate of S6K (Fig. 7B and C). These results indicated that largazole suppresses mTORC1 signaling. mTOR forms two structurally and functionally 


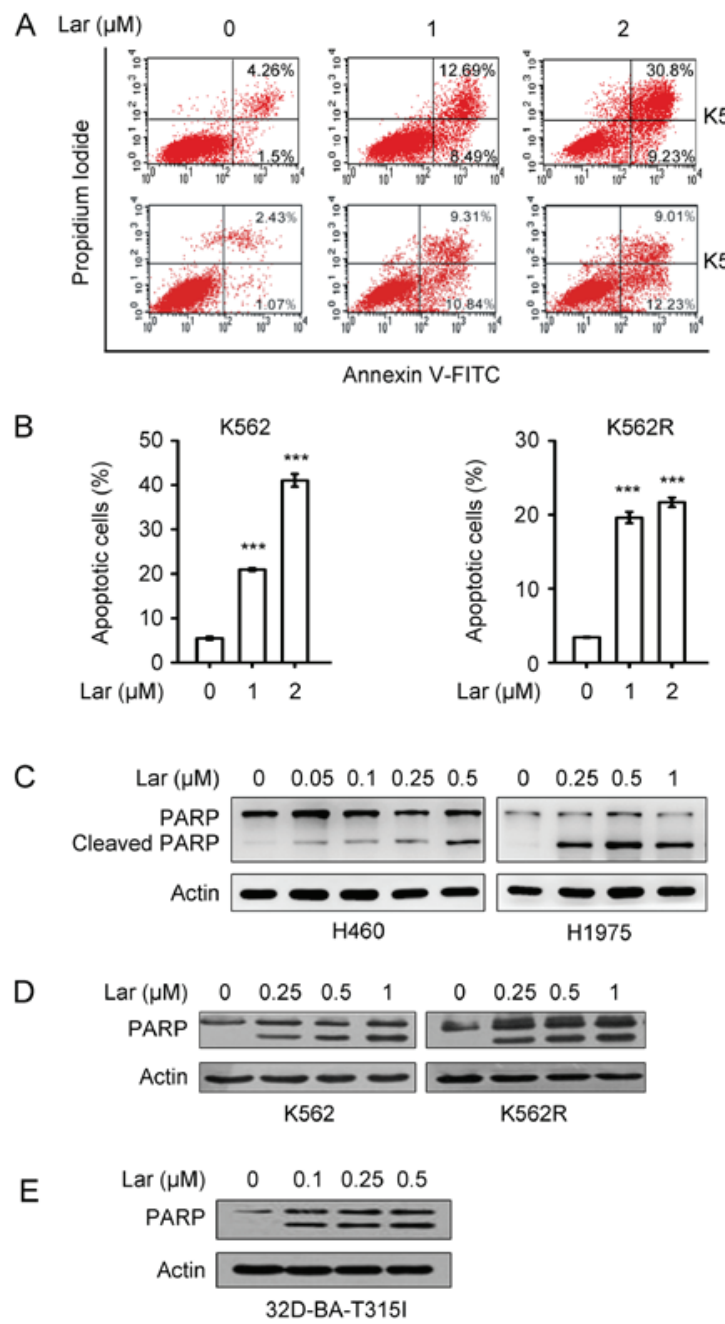

Figure 6. Largazole induces cancer cell apoptosis. (A) CML cells were treated with largazole at 1 and $2 \mu \mathrm{M}$ for $48 \mathrm{~h}$, and cell apoptosis was analyzed by flow cytometry using Annexin V-FITC/propidium iodide staining. Representative images are shown. Repeated number, $\mathrm{n}=3$. (B) Quantification of flow cytometry analysis of apoptosis. ${ }^{* * * *} \mathrm{P}<0.001$, difference vs. control group. Repeated number, $\mathrm{n}=3$. (C-E) NSCLC and CML cells were treated with the indicated concentrations of largazole for $24 \mathrm{~h}$, and whole-cell lysates were subjected to western blot analysis with the indicated antibodies. Actin was used as a loading control. CML, chronic myeloid leukemia; NSCLC, non-small cell lung cancer.
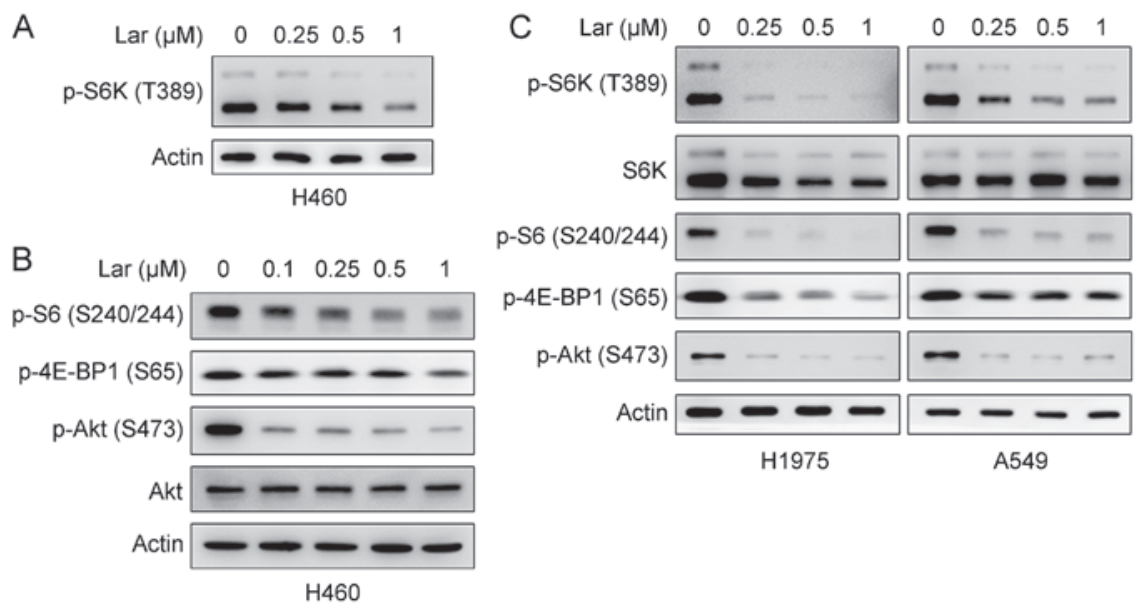

Figure 7. Largazole inhibits the mTOR signaling pathway downstream of MSI2. (A-C) NSCLC cells were treated with the indicated concentrations of largazole for $24 \mathrm{~h}$, harvested, and subjected to western blot analysis using the indicated antibodies. Actin was used as a loading control. Repeated number, $\mathrm{n}=3$. NSCLC, non-small cell lung cancer; mTOR, mammalian target of rapamycin; MSI2, Musashi-2.

distinct multi-protein complexes, mTORC1 and mTORC2 (39). mTORC2 phosphorylates Akt on S473 (40). It was then investigated whether largazole exerted an inhibitory effect on the mTORC2 signaling pathway. In all tested cells, 
largazole markedly suppressed the phosphorylation of Akt (S473), indicating that largazole inhibits mTORC2 signaling (Fig. 7B and C). The aforementioned results suggest that largazole represses cell proliferation, at least in part, by targeting MSI2 to suppress mTOR signaling pathway.

\section{Discussion}

Accumulating evidence indicates that MSI2 plays a key role in cell proliferation, cancer stemness, epithelial-to-mesenchymal transition, invasion, migration and drug resistance in multiple types of cancer $(12,41)$. The MSI2 level is increased in various types of tumors, and its overexpression is closely associated with aggressive characteristics and poor prognosis (12-14,20). We herein demonstrated that the protein levels of MSI2 were significantly increased in lung cancer tissues from 6 Chinese patients compared with those in adjacent normal lung tissues (Fig. 1C and D). An immunohistochemistry analysis of matched NSCLC specimens containing normal lung tissue, primary tumor and tumor-positive lymph nodes from 14 American patients demonstrated a significant increase of MSI2 levels in the primary tumor (2.4-fold) and in the lymph nodes (4.5-fold) compared with normal lung tissue (16). At the mRNA level, the expression of MSI2 was inversely associated with clinical outcome (Fig. 1G). These data suggest a crucial role of MSI 2 in lung carcinogenesis.

The key role of MSI2 in diverse cancers has prompted an attempt to develop small-molecule inhibitors of this protein. Using molecular docking analysis, largazole was identified as a novel compound that was able to bind to human MSI2 (Fig. 2). The homology between human MSI2 and mus musculus MSI2 is $94.22 \%$, while the key binding sites of human MSI2 for largazole are identical to those in mouse MSI2, suggesting that largazole may also bind and inhibit mus musculus MSI2. Furthermore, the homology between human MSI2 and MSI1 is 75\% (12), and the amino acids of the key sites displayed by the docking analysis are also found in MSI1; thus, largazole may also bind MSI1. This possibility warrants further investigation. Largazole drastically decreased the protein levels of MSI2 in multiple types of cancer cells, including H460, H1975, A549, K562 and K562R, among others (Fig. 3), indicating that largazole-induced downregulation of MSI2 is not a cell type-dependent event. It has been reported that MSI2 directly interacts with the tumor suppressor deleted in breast cancer-2, and this interaction promotes polyubiquitination-mediated proteasomal degradation of MSI2 in breast cancer (42). Ubiquitin-specific protease 10 can interact with MSI2 and regulate MSI2 stability via deubiquitination in colon cancer (43). MSI2 is a direct transcriptional target of Kruppel-like factor 4, a zinc finger transcription factor, in pancreatic ductal adenocarcinomas (20). Transcription factors USF2 and PLAG1 bind the promoter of MSI2 and promote its transcription thus collectively playing a key role in hematopoietic stem and progenitor cell function (44). These findings suggest that the MSI2 protein level may be regulated at the transcriptional and/or post-translational level. Of note, pretreatment with the proteasome inhibitor PS-341 or the lysosome inhibitor CQ were unable to block largazole-induced downregulation of MSI2 in H460 and K562 cells (Fig. 4A-D). Moreover, largazole markedly reduced the mRNA levels of MSI2 in H460, H1975 and K562 cells (Fig. 4E-G). The results mentioned above suggest that largazole did not induce protein degradation of MSI2 by the ubiquitin-proteasome pathway or by the lysosome-dependent pathway, but markedly reduced MSI2 mRNA. Largazole may also inhibit MSI2 function by direct binding via hydrogen bonds, thereby inhibiting interactions between MSI2 and its cofactors. This possibility should be tested in future studies.

Although the targets of the MSI2 protein have not been fully identified, studies have shown that MSI2 regulates numerous oncogenic pathways involved in cell cycle progression, proliferation and metabolism, among others $(12,45)$. For example, MSI2 was found to provide essential support for transforming growth factor- $\beta$ signaling and inhibit tight junction-associated claudins to promote NSCLC metastasis (16). MSI2 has been shown to act as an oncoprotein through mTORC1 activation in colorectal cancer $(15,35)$. MSI2 promotes invasion and migration through activation of the JAK2/STAT3 signaling pathway in bladder cancer (23). Thus, it was inferred that blocking MSI2 function with small-molecule inhibitors may be of therapeutic value in various malignancies. Indeed, the MSI2-targeting compound largazole significantly suppressed the proliferation of cancer cells and induced apoptosis (Figs. 5A-D and 6). Of note, largazole markedly inhibited the proliferation of mononuclear cells that were isolated from the bone marrow of 5 patients with CML (Fig. 5E). Largazole exerted potent growth-inhibitory effects in multiple cancer cell models, whereas non-transformed epithelial cells survived at higher doses $(34,46,47)$. In a human colon cancer HCT116 xenograft mouse model, largazole significantly inhibited tumor growth with no obvious toxicity $(29,48)$, indicating that largazole has high bioavailability and low toxicity. Further studies demonstrated that largazole drastically suppressed mTORC1, which was found to be downstream of MSI2, and repressed mTORC2 signaling in NSCLC cell lines (Fig. 7). Taken together, these findings suggest that largazole inhibits cell proliferation, at least in part, by targeting MSI2 to suppress mTOR signaling, and may hold promise as a candidate for cancer therapy.

\section{Acknowledgements}

The authors would like to thank Professor Warren Pear of University of Pennsylvania for kindly providing the MSCV-Bcr-Abl-T315I-IRES/GFP retroviral transducing vector, and Professor Brian Druker of Oregon Health \& Science University for providing the pSRalpha plasmids containing the wild-type and T315I mutant Bcr-Abl.

\section{Funding}

The present study was supported by grants from the National Key Research and Development Program of China (no. 2016YFC0905501), the National Natural Science Funds for Distinguished Young Scientists (no. 81425025), the Key Project of the National Natural Science Foundation of China (no. 81830093), the CAMS Innovation Fund for Medical Sciences (CIFMS; no. 2019-I2M-1-003), and the National Natural Science Foundation of China (nos. 81672765 and 
81802796). The study sponsors had no role in the design of the study, the data collection, analysis, or interpretation, the writing of the article, or the decision to submit for publication.

\section{Availability of data and materials}

The data generated or analyzed during the present study are included in this published article or are available from the corresponding author on reasonable request.

\section{Authors' contributions}

The project was conceived and designed by GBZ. The experiments were conducted by MW, XYS, KJZ and GZW. Biospecimens were harvested/provided by YCZ, YCH and SJ. Data were analyzed by GBZ, JL and YZL. The manuscript was written by GBZ. All the authors have read and approved the final version of the manuscript.

\section{Ethics approval and consent to participate}

The present study was approved by the Institutional Review Board of the Institute of Zoology, Chinese Academy of Sciences and the Third Affiliated Hospital of Kunming Medical University; all tissue samples were obtained with written informed consent from the patients or their families.

\section{Patient consent for publication}

Not applicable.

\section{Competing interests}

The authors declare that they have no competing interests.

\section{References}

1. Bray F, Ferlay J, Soerjomataram I, Siegel RL, Torre LA and Jemal A: Global cancer statistics 2018: GLOBOCAN estimates of incidence and mortality worldwide for 36 cancers in 185 countries. CA Cancer J Clin 68: 394-424, 2018.

2. Herbst RS, Heymach JV and Lippman SM: Lung cancer. N Engl J Med 359: 1367-1380, 2008.

3. Herbst RS, Morgensztern D and Boshoff C: The biology and management of non-small cell lung cancer. Nature 553: 446-454, 2018.

4. Hirsch FR, Scagliotti GV, Mulshine JL, Kwon R, Curran WJ Jr, Wu YL and Paz-Ares L: Lung cancer: Current therapies and new targeted treatments. Lancet 389: 299-311, 2017.

5. Siegel RL, Miller KD and Jemal A: Cancer statistics, 2019. CA Cancer J Clin 69: 7-34, 2019.

6. Zhou G: Tobacco, air pollution, environmental carcinogenesis, and thoughts on conquering strategies of lung cancer. Cancer Biol Med 16: 700-713, 2019.

7. Anji A and Kumari M: Guardian of Genetic Messenger-RNABinding Proteins. Biomolecules 6: 4, 2016.

8. Pereira B, Billaud M and Almeida R: RNA-Binding Proteins in Cancer: Old Players and New Actors. Trends Cancer 3: 506-528, 2017.

9. Fox RG, Park FD, Koechlein CS, Kritzik M and Reya T: Musashi signaling in stem cells and cancer. Annu Rev Cell Dev Biol 31: 249-267, 2015.

10. Lan L, Xing M, Douglas JT, Gao P, Hanzlik RP and Xu L: Human oncoprotein Musashi-2 N-terminal RNA recognition motif backbone assignment and identification of RNA-binding pocket. Oncotarget 8: 106587-106597, 2017.
11. Liu Y, Fan Y, Wang X, Huang Z, Shi K and Zhou B: Musashi-2 is a prognostic marker for the survival of patients with cervical cancer. Oncol Lett 15: 5425-5432, 2018.

12. Kudinov AE, Karanicolas J, Golemis EA and Boumber Y: Musashi RNA-Binding Proteins as Cancer Drivers and Novel Therapeutic Targets. Clin Cancer Res 23: 2143-2153, 2017.

13. Ito T, Kwon HY, Zimdahl B, Congdon KL, Blum J, Lento WE, Zhao C, Lagoo A, Gerrard G, Foroni L, et al: Regulation of myeloid leukaemia by the cell-fate determinant Musashi. Nature 466: 765-768, 2010.

14. Kharas MG, Lengner CJ, Al-Shahrour F, Bullinger L, Ball B, Zaidi S, Morgan K, Tam W, Paktinat M, Okabe R, et al: Musashi-2 regulates normal hematopoiesis and promotes aggressive myeloid leukemia. Nat Med 16: 903-908, 2010.

15. Wang S, Li N, Yousefi M, Nakauka-Ddamba A, Li F, Parada K, Rao S, Minuesa G, Katz Y, Gregory BD, et al: Transformation of the intestinal epithelium by the MSI2 RNA-binding protein. Nat Commun 6: 6517, 2015.

16. Kudinov AE, Deneka A, Nikonova AS, Beck TN, Ahn YH, Liu X, Martinez CF, Schultz FA, Reynolds S, Yang DH, et al: Musashi-2 (MSI2) supports TGF- $\beta$ signaling and inhibits claudins to promote non-small cell lung cancer (NSCLC) metastasis. Proc Natl Acad Sci USA 113: 6955-6960, 2016

17. Zhang MR, Xi S, Shukla V, Hong JA, Chen H, Xiong Y, Ripley RT, Hoang CD and Schrump DS: The Pluripotency Factor Musashi-2 Is a Novel Target for Lung Cancer Therapy. Ann Am Thorac Soc 15 (Suppl 2): S124, 2018.

18. Kang MH, Jeong KJ, Kim WY, Lee HJ, Gong G, Suh N Győrffy B, Kim S, Jeong SY, Mills GB, et al: Musashi RNA-binding protein 2 regulates estrogen receptor 1 function in breast cancer. Oncogene 36: 1745-1752, 2017.

19. Dong P, Xiong Y, Hanley SJB, Yue J and Watari H: Musashi-2, a novel oncoprotein promoting cervical cancer cell growth and invasion, is negatively regulated by p53-induced miR-143 and miR-107 activation. J Exp Clin Cancer Res 36: 150, 2017.

20. Guo K, Cui J, Quan M, Xie D, Jia Z, Wei D, Wang L, Gao Y, Ma Q and Xie K: The Novel KLF4/MSI2 Signaling Pathway Regulates Growth and Metastasis of Pancreatic Cancer. Clin Cancer Res 23: 687-696, 2017.

21. Li Z, Jin H, Mao G, Wu L and Guo Q: Msi2 plays a carcinogenic role in esophageal squamous cell carcinoma via regulation of the Wnt/ $\beta$-catenin and Hedgehog signaling pathways. Exp Cell Res 361: 170-177, 2017.

22. He L, Zhou X, Qu C, Hu L, Tang Y, Zhang Q, Liang M and Hong J: Musashi 2 predicts poor prognosis and invasion in hepatocellular carcinoma by driving epithelial-mesenchymal transition. J Cell Mol Med 18: 49-58, 2014.

23. Yang C, Zhang W, Wang L, Kazobinka G, Han X, Li B and Hou T: Musashi-2 promotes migration and invasion in bladder cancer via activation of the JAK2/STAT3 pathway. Lab Invest 96: 950-958, 2016

24. Lee J, An S, Choi YM, Lee J, Ahn KJ, Lee JH, Kim TJ, An IS and Bae S: Musashi-2 is a novel regulator of paclitaxel sensitivity in ovarian cancer cells. Int J Oncol 49: 1945-1952, 2016.

25. Zhang H, Tan S, Wang J, Chen S, Quan J, Xian J, Zhang S, He J and Zhang L: Musashi2 modulates K562 leukemic cell proliferation and apoptosis involving the MAPK pathway. Exp Cell Res 320: 119-127, 2014

26. Han Y, Ye A, Zhang Y, Cai Z, Wang W, Sun L, Jiang S, Wu J, Yu K and Zhang S: Musashi-2 Silencing Exerts Potent Activity against Acute Myeloid Leukemia and Enhances Chemosensitivity to Daunorubicin. PLoS One 10: e0136484, 2015.

27. Lan L, Liu H, Smith AR, Appelman C, Yu J, Larsen S, Marquez RT, Wu X, Liu FY, Gao P, et al: Natural product derivative Gossypolone inhibits Musashi family of RNA-binding proteins. BMC Cancer 18: 809, 2018.

28. Minuesa G, Albanese SK, Xie W, Kazansky Y, Worroll D, Chow A, Schurer A, Park SM, Rotsides CZ, Taggart J, et al: Small-molecule targeting of MUSASHI RNA-binding activity in acute myeloid leukemia. Nat Commun 10: 2691, 2019.

29. Hong J and Luesch H: Largazole: From discovery to broad-spectrum therapy. Nat Prod Rep 29: 449-456, 2012.

30. Győrffy B, Surowiak P, Budczies J and Lánczky A: Online survival analysis software to assess the prognostic value of biomarkers using transcriptomic data in non-small-cell lung cancer. PLoS One 8: e82241, 2013.

31. Zeng X, Yin B, Hu Z, Liao C, Liu J, Li S, Li Z, Nicklaus MC, Zhou $\mathrm{G}$ and Jiang S: Total synthesis and biological evaluation of largazole and derivatives with promising selectivity for cancers cells. Org Lett 12: 1368-1371, 2010. 
32. Hu Z, Pan XF, Wu FQ, Ma LY, Liu DP, Liu Y, Feng TT, Meng FY, Liu XL, Jiang QL, et al: Synergy between proteasome inhibitors and imatinib mesylate in chronic myeloid leukemia. PLoS One 4: e6257, 2009.

33. Taori K, Paul VJ and Luesch H: Structure and activity of largazole, a potent antiproliferative agent from the Floridian marine cyanobacterium Symploca sp. J Am Chem Soc 130: 1806-1807, 2008.

34. Wu LC, Wen ZS, Qiu YT, Chen XQ, Chen HB, Wei MM, Liu Z, Jiang S and Zhou GB: Largazole Arrests Cell Cycle at G1 Phase and Triggers Proteasomal Degradation of E2F1 in Lung Cancer Cells. ACS Med Chem Lett 4: 921-926, 2013.

35. Li N, Yousefi M, Nakauka-Ddamba A, Li F, Vandivier L, Parada K, Woo DH, Wang S, Naqvi AS, Rao S, et al: The Msi Family of RNA-Binding Proteins Function Redundantly as Intestinal Oncoproteins. Cell Rep 13: 2440-2455, 2015.

36. Laplante M and Sabatini DM: mTOR signaling in growth control and disease. Cell 149: 274-293, 2012.

37. Saxton RA and Sabatini DM: mTOR Signaling in Growth, Metabolism, and Disease. Cell 169: 361-371, 2017.

38. Shimobayashi $M$ and Hall MN: Making new contacts: The mTOR network in metabolism and signalling crosstalk. Nat Rev Mol Cell Biol 15: 155-162, 2014.

39. Zoncu R, Efeyan A and Sabatini DM: mTOR: From growth signal integration to cancer, diabetes and ageing. Nat Rev Mol Cell Biol 12: 21-35, 2011.

40. Sarbassov DD, Guertin DA, Ali SM and Sabatini DM: Phosphorylation and regulation of Akt/PKB by the rictor-mTOR complex. Science 307: 1098-1101, 2005.

41. Fang T, Lv H, Wu F, Wang C, Li T, Lv G, Tang L, Guo L, Tang S, Cao D, et al: Musashi 2 contributes to the stemness and chemoresistance of liver cancer stem cells via LIN28A activation. Cancer Lett 384: 50-59, 2017.
42. Choi YM, Kim KB, Lee JH, Chun YK, An IS, An S and Bae S: DBC2/RhoBTB2 functions as a tumor suppressor protein via Musashi-2 ubiquitination in breast cancer. Oncogene 36: 2802-2812, 2017

43. Ouyang SW, Liu TT, Liu XS, Zhu FX, Zhu FM, Liu XN and Peng ZH: USP10 regulates Musashi-2 stability via deubiquitination and promotes tumour proliferation in colon cancer. FEBS Lett 593: 406-413, 2019.

44. Belew MS, Bhatia S, Keyvani Chahi A, Rentas S, Draper JS and Hope KJ: PLAG1 and USF2 Co-regulate Expression of Musashi-2 in Human Hematopoietic Stem and Progenitor Cells. Stem Cell Reports 10: 1384-1397, 2018.

45. Minuesa G, Antczak C, Shum D, Radu C, Bhinder B, Li Y, Djaballah H and Kharas MG: A 1536-well fluorescence polarization assay to screen for modulators of the MUSASHI family of RNA-binding proteins. Comb Chem High Throughput Screen 17: 596-609, 2014.

46. Schnekenburger M, Dicato M and Diederich M: Epigenetic modulators from "The Big Blue": A treasure to fight against cancer. Cancer Lett 351: 182-197, 2014.

47. Pilon JL, Clausen DJ, Hansen RJ, Lunghofer PJ, Charles B, Rose BJ, Thamm DH, Gustafson DL, Bradner JE and Williams RM: Comparative pharmacokinetic properties and antitumor activity of the marine HDACi Largazole and Largazole peptide isostere. Cancer Chemother Pharmacol 75: 671-682, 2015.

48. Liu Y, Salvador LA, Byeon S, Ying Y, Kwan JC, Law BK, Hong $\mathrm{J}$ and Luesch $\mathrm{H}$ : Anticolon cancer activity of largazole, a marine-derived tunable histone deacetylase inhibitor. J Pharmacol Exp Ther 335: 351-361, 2010. 